\title{
Efficient and Effective Handling of Exceptions in Java Points-to Analysis
}

\author{
George Kastrinis and Yannis Smaragdakis \\ Dept. of Informatics, University of Athens, Greece \\ \{gkastrinis, smaragd\}@di.uoa.gr
}

\begin{abstract}
A joint points-to and exception analysis has been shown to yield benefits in both precision and performance. Treating exceptions as regular objects, however, incurs significant and rather unexpected overhead. We show that in a typical joint analysis most of the objects computed to flow in and out of a method are due to exceptional control-flow and not normal call-return control-flow. For instance, a context-insensitive analysis of the Antlr benchmark from the DaCapo suite computes 4-5 times more objects going in or out of a method due to exceptional control-flow than due to normal control-flow. As a consequence, the analysis spends a large amount of its time considering exceptions.

We show that the problem can be addressed both effectively and elegantly by coarsening the representation of exception objects. An interesting find is that, instead of recording each distinct exception object, we can collapse all exceptions of the same type, and use one representative object per type, to yield nearly identical precision (loss of less than $0.1 \%$ ) but with a boost in performance of at least $50 \%$ for most analyses and benchmarks and large space savings (usually $40 \%$ or more).
\end{abstract}

\section{Introduction}

Points-to analysis is a fundamental static program analysis. It consists of computing a static abstraction of all the data that a pointer variable (and, by extension, any pointer expression) can point to during program execution. Points-to analysis is often the basis of most other higher-level client analyses (e.g., may-happen-in-parallel, static cast elimination, escape analysis, and more). It is also inter-related with call-graph construction, since the values of a pointer determine the target of dynamically resolved calls, such as object-oriented dynamically dispatched method calls or functional lambda applications.

An important question regarding points-to analysis (as well as client analyses based on it) concerns the handling of exceptions, in languages that support exception-based control flow. The emphasis of our work is on Java- a common target of points-to analysis work-but similar ideas are likely to apply to other languages, such as $\mathrm{C \#}$ and $\mathrm{C}++$. This is an important topic because exceptional control flow cannot be ignored for several client analysis (e.g., information leak or other security analyses) and if handled crudely it can destroy the precision of the base points-to analysis.

In the past, most practical points-to analysis algorithms have relied on conservative approximations of exception handling [19,20]. The well-known points-to analysis libraries SPARK [19] and PADDLE [18] both model exception throwing as an assignment

K. De Bosschere and R. Jhala (Eds.): CC 2013, LNCS 7791, pp. 41-60, 2013.

(C) Springer-Verlag Berlin Heidelberg 2013 
to a single global variable for all exceptions thrown in a program. The variable is then read at the site of an exception catch. This approach is sound but highly imprecise because it ignores the information about what exceptions can propagate to a catch site. For clients that care about exception objects specifically (e.g., computing which throw statement can reach which catch clause), precise exception handling has been added on top of a base points-to analysis [7- 9]. Fu and Ryder's "exception-chain analysis" [8] is representative. It works on top of SPARK, with its conservative modeling of exceptions, but then performs a very precise analysis of the flow of exception objects. However, this approach has a high computational overhead. Furthermore, the approach does not recover the precision lost for the base points-to results for objects that do not represent exceptions.

Based on the above, the Doop framework [2] (which is also the context of our work) has introduced a joint points-to and exception analysis [1]. Doop expresses exceptionanalysis logic in modular rules, mutually recursive with the points-to analysis logic: Exception handling can cause variables to point to objects (of an exception type), can make code reachable, etc. Points-to results are, in turn, used to compute what objects are thrown at a throw statement. The exception analysis logic on its own is "as precise as can be" as it fully models the Java semantics for exceptions. Approximation is only introduced due to the static abstractions used for contexts and objects in the points-to analysis. Thus, exception analysis is specified in a form that applies to points-to analyses of varying precision, and the exception analysis transparently inherits the points-to analysis precision. The result is an analysis that achieves very high precision and performance for points-to results, while also matching the precision of techniques specifically for exception-related queries, as in the Fu and Ryder exception-chain analysis.

The motivation for our work is that, despite the benefits of the Doop approach, there is significant room for improvement. The joint points-to and exception analysis performs heavy work for exception objects alone. An indicative metric is the following: Consider the number of objects pointed to by method parameters or its return value vs. the objects thrown and not caught by the current method or by methods called by it. The former number represents the objects that flow into or out of each method due to normal control-flow, while the latter shows the objects that flow out of the method due to exceptions. Our experiments show that the latter number is often several times larger than the former. (We present full results later.) This is counterintuitive and suggests that the analysis performs unexpectedly much work on exceptions alone.

To address this issue we observe that most client analyses do not care about exception objects specifically. They do, however, care about the impact of exceptions to the rest of the points-to and call-graph facts. For instance, the effectiveness of a client analysis such as static cast elimination is not impacted in practice by the few optimization opportunities that lie inside exception handlers or that involve objects of an exception type. But the analysis $i s$ impacted by code possibly executed only because of exception handling, or variables that point to extra objects as a result of an exception handler's execution. In other words, we would like precise handling of exceptions only to the extent that they impact the precision of the base points-to analysis, even if the information over exception objects themselves is less precise. (Note that this is very different from the SPARK or PADDLE handling of all exceptions through a single global variable: That approach does 
adversely impact the precision and performance of the base analysis-e.g., it more than doubles the number of edges of a context-sensitive call-graph [1, Fig.12]).

Therefore, our approach consists of coarsening the representation of exception objects in two ways. First, we treat exception objects context-insensitively, even for an otherwise context-sensitive analysis 1 Second, we merge exception objects and represent them as a single object per-dynamic-type. The per-type treatment is important for maintaining precision, since the main purpose of an exception object is to trigger appropriate exception handling code (i.e., a catch clause keyed on the type of the object caught).

We find that this approach is both easy to specify and implement, as well as highly effective. For instance, for a 1-object-sensitive analysis we obtain a $60 \%$ average speedup for the "antlr" benchmark and a $225 \%$ average speedup for the "eclipse" benchmark of the DaCapo suite (with similar speedups for other benchmarks) just by employing the "merge exception objects per-type" idea. This speedup is accompanied by significant space savings in the analysis. Crucially, the performance increase does not entail any loss of precision for results unrelated to exception objects. All precision metrics of the analysis remain virtually identical. Namely, the numbers of call-graph nodes and edges, methods that can be successfully devirtualized, and casts that can be statically eliminated remain the same up to at least three significant digits.

In summary, the contributions of our work are as follows:

- We give a concise and general model of flow-insensitive, context- and field-sensitive points-to analyses and call-graph construction for a language with exceptions. Although a joint exception and points-to analysis has been formulated before [1], it was expressed by-example. In contrast, we give a small, closed set of rules and definitions of input domains. That is, we present all the relevant detail of the analysis in a closed form, assuming a simplified intermediate language as input.

- We present measurements demonstrating that the impact of exceptions on points-to analysis performance metrics is significant. A points-to analysis that tries to model exceptions precisely ends up spending much of its time and space computing results for exception-based control-flow.

- We define on top of our model two simple ways to coarsen the representation of exception objects without affecting any other aspect of the points-to or exception logic.

- We show that our approach is very effective in practice, yielding both significant speedup and space savings. Our technique is the default in the upcoming version of the Doop framework as it gains performance without adversely impacting precision.

In the following sections we define an abstraction of context-sensitive points-to analysis and enhance it with exception handling logic (Section 2), present our technique in this abstract model (Section 3), detail its performance in a series of experiments (Section 4), and discuss related work in more detail (Section 5).

\footnotetext{
${ }^{1}$ Context-sensitivity is a general approach that achieves tractable and usefully high precision in points-to analyis. It consists of qualifying local program variables, and possibly (heap) object abstractions, with context information: the analysis collapses information (e.g., "what objects this method argument can point to") over all possible executions that result in the same context, while separating all information for different contexts.
} 


\section{Background: Model of Points-to Analysis}

We next present a model of context-sensitive, flow-insensitive points-to analysis algorithms, as well as the enhancement of the model for computing exception information in mutual recursion with the analysis. Interestingly, the logical formalism that we use in our model is quite close to the actual implementation of the analysis in the Doop framework, under simplifications and omissions that we describe.

\subsection{Base Points-to Analysis}

We model a wide range of flow-insensitive points-to analyses together with the associated call-graph computation as a set of customizable Datalog rules, i.e., monotonic logical inferences that repeatedly apply to infer more facts until fixpoint. Our rules do not use negation in a recursive cycle, or other non-monotonic logic constructs, resulting in a declarative specification: the order of evaluation of rules or examination of clauses cannot affect the final result. The same abstract model applies to a wealth of analyses. We use it to model a context-insensitive Andersen-style analysis, as well as several context-sensitive analyses, both call-site-sensitive [25, 26] and object-sensitive [23].

The input language is a simplified intermediate language with a) a "new" instruction for allocating an object; b) a "move" instruction for copying between local variables; c) "store" and "load" instructions for writing to the heap (i.e., to object fields); d) a "virtual method call" instruction that calls the method of the appropriate signature that is defined in the dynamic class of the receiver object. This language models well the Java bytecode representation, but also other high-level intermediate languages. (It does not, however, model languages such as $\mathrm{C}$ or $\mathrm{C}++$ that can create pointers through an address-of operator. The techniques used in that space are fairly different-e.g., [12, 13.) The specification of our points-to analysis as well as the input language are in line with those in the work of others [10,21], although we also integrate elements such as on-the-fly call-graph construction and field-sensitivity.

Specifying the analysis logically as Datalog rules has the advantage that the specification is close to the actual implementation. Datalog has been the basis of several implementations of program analyses, both low-level [2, 17, 24, 29,30] and high-level [5, 11]. Indeed, the analysis we show is a faithful model of the implementation in the Doop framework [2]. Our specification of the analysis (Figures 1, 2) is an abstraction of the actual implementation in the following ways:

- The implementation has many more rules. It covers the full complexity of the language, including rules for handling reflection, native methods, static calls and fields, string constants, implicit initialization, threads, and a lot more. The Doop implementation currently contains over 600 rules in the common core of all analyses, as opposed to the dozen-or-so rules we examine here. (Note, however, that these dozen rules are the most crucial for points-to analysis. They also correspond fairly closely to the algorithms specified in other formalizations of points-to analyses in the literature [22, 28].)

- The implementation also reflects considerations for efficient execution. The most important is that of defining indexes for the key relations of the evaluation. Furthermore, it designates some relations as functions, defines storage models for relations 


\begin{tabular}{|c|c|c|}
\hline$V$ is a set of variables & \multicolumn{2}{|c|}{$H$ is a set of heap abstractions } \\
\hline$M$ is a set of methods & \multicolumn{2}{|c|}{$S$ is a set of method signatures (including name) } \\
\hline$F$ is a set of fields & \multicolumn{2}{|c|}{$I$ is a set of instructions (e.g., invocation sites) } \\
\hline$T$ is a set of class types & \multicolumn{2}{|c|}{$\mathbb{N}$ is the set of natural numbers } \\
\hline \multirow{2}{*}{\multicolumn{3}{|c|}{$\begin{array}{l}H C \text { is a set of heap contexts } C \text { is a set of contexts } \\
\text { ALLOC (var:V heap }: H, \text { meth }: M \text { ) FoRMALARG }(n\end{array}$}} \\
\hline & & \\
\hline & \multicolumn{2}{|c|}{ Move (to : $V$, from $: V)$} \\
\hline LOAD (to : V, base $: V$, fld $: F)$ & & $($ meth $: M$, ret $: V)$ \\
\hline \multicolumn{2}{|l|}{ StoRe (base : V, fld : F, from : V } & $\mathrm{N}($ invo $: I$, var $: V)$ \\
\hline \multicolumn{2}{|l|}{ VCALL (base : V, sig : S, invo :I) } & , this \\
\hline \multicolumn{2}{|l|}{ HEAPTYPE (heap $: H$, type $: T$ ) } & LоокUр (type : $T$, sig $: S$, meth $: M$ ) \\
\hline \multicolumn{3}{|l|}{ INMETHOD (instr $: I$, meth $: M$ ) } \\
\hline \multicolumn{3}{|c|}{ VARPoInTSTo (var : V, ctx : C, heap :H, hctx :HC) } \\
\hline \multicolumn{3}{|c|}{ CaLLGRAPH (invo : I, callerCtx : $C$, meth $: M$, calleeCtx $: C$ ) } \\
\hline \multicolumn{3}{|c|}{ FLDPoInTsTo (baseH : H, baseHCtx :HC, fld :F, heap :H, hctx :HC) } \\
\hline \multicolumn{3}{|c|}{ InTERProcAssign (to $: V$, to Ctx $: C$, from $: V$, from $C t x: C$ ) } \\
\hline \multicolumn{3}{|l|}{ ReAchable (meth $: M, c t x: C)$} \\
\hline \multicolumn{3}{|c|}{ RECORD (heap $: H$, ctx $: C)=$ newHCtx $: \boldsymbol{H C}$} \\
\hline \multicolumn{3}{|c|}{ Merge (heap : H, hctx :HC, invo $: I$, ctx $: C)=$ newCtx : } \\
\hline
\end{tabular}

Fig. 1. Our domain, input relations, output relations, and constructors of contexts

(e.g., how many bits each variable uses), designates intermediate relations as "materialized views" or not, etc.

Figure 1 shows the domain of our analysis (i.e., the different sets that comprise the space of our computation), its input relations, the intermediate and output relations, as well as two constructor functions, responsible for producing new objects that represent contexts. We explain some of these components below:

- The input relations are standard and correspond to the intermediate language for our analysis. For instance, the AlLoc relation represents every instruction that allocates a new heap object, heap, and assigns it to local variable var inside method meth. (Note that every local variable is defined in a unique method, hence the meth argument is also implied by var but included for conciseness of later rules.) There are input relations for each instruction type (Move, LOAD, STORE and VCALL) as well as input relations encoding the type system and symbol table information. For instance, LookUp matches a method signature to the actual method definition inside a type.

- The main output relations of our points-to analysis and call-graph computation are VARPointsTo and CallGraph. The VarPointsTo relation links a variable (var) to a heap object (heap). (A heap object is identified by its allocation site.) Both the variable and the heap object are qualified by "context" elements in our analysis: a plain context for the variable and a heap context for the heap object. Similarly, the CALLGRAPH relation qualifies both its source (an invocation site) and its target (a method) with contexts. Other intermediate relations (FldPointsTo, InterProcAssign, Reachable) correspond to standard concepts and are introduced for conciseness and readability.

- The base rules are not concerned with what kind of context-sensitivity is used. The same rules can be used for a context-insensitive analysis (by only ever creating a single context object), for a call-site-sensitive analysis, or for an object-sensitive analysis, for 
any context depth. These aspects are completely hidden behind constructor functions RECORD and MERGE, following the usage and naming convention of earlier work [28]. RECORD takes all available information at the allocation site of an object and combines it to produce a new heap context, while MERGE takes all available information at the call site of a method and combines it to create a new context. (Hence, the name "Merge" refers to merging contexts and is unrelated to the idea of merging exception objects pertype, which we discuss later in this paper.) These functions are sufficient for modeling a very large variety of context-sensitive analyses 2 Note that the use of such constructors is not part of regular Datalog and can result in infinite structures (e.g., one can express unbounded call-site sensitivity) if care is not taken.

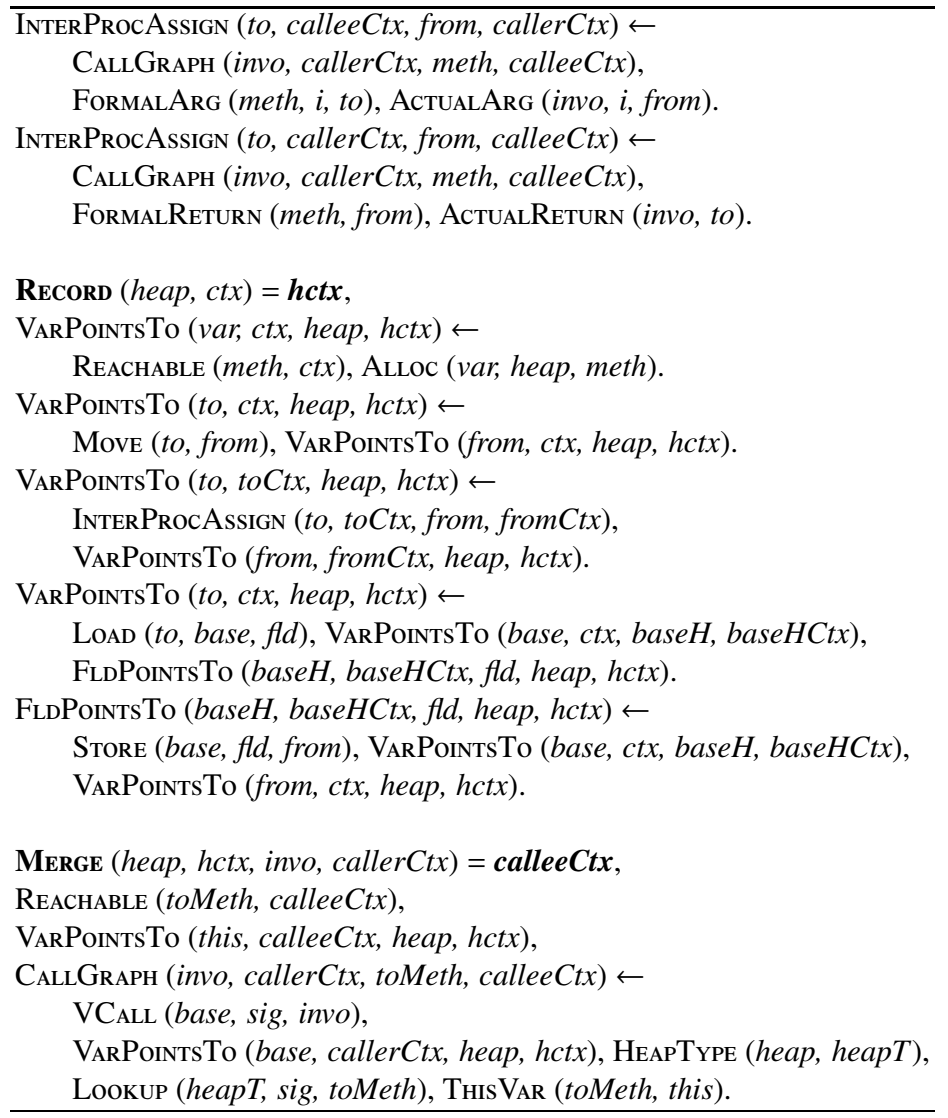

Fig. 2. Datalog rules for the points-to analysis and call-graph construction

${ }^{2}$ Explaining the different kinds of context-sensitivity produced by varying RECORD and MerGE is beyond the scope of this paper but is fully covered in past literature [28]. To give a single example, however, a 1-call-site-sensitive analysis with a context-sensitive heap has $C=H C=$ $I$ (i.e., both the context and the heap context are a single instruction), RECoRD (heap, $c t x)=$ ctx and Merge (heap, hctx, invo, callerCtx) = invo. That is, when an object is allocated, its (heap) context is that of the allocating method, and when a method is called, its context is its call-site. 
Figure 2 shows the points-to analysis and call-graph computation. The rule syntax is simple: the left arrow symbol $(\leftarrow)$ separates the inferred fact (i.e., the head of the rule) from the previously established facts (i.e., the body of the rule). For instance, the very last rule says that if the original program has an instruction making a virtual method call over local variable base (this is an input fact), and the computation so far has established that base can point to heap object heap, then the called method is looked up inside the type of heap and several further facts are inferred: that the looked up method is reachable, that it has an edge in the call-graph from the current invocation site, and that its this variable can point to heap. Additionally, the Merge function is used to possibly create (or look up) the right context for the current invocation.

\subsection{Adding Exceptions}

We can now easily add exception handling to our input language and express a precise exception analysis via rules that are mutually recursive with the base points-to analysis rules. The algorithm is essentially that of [1] but stated more concisely: we hide exception handler lookup details by assuming a more sophisticated input relation $\mathrm{CATCH}_{\text {. }}$

\begin{tabular}{l}
$\frac{\text { Throw (instr }: I, e: V)}{\text { CATCH }(\text { heapT }: T \text {, instr }: I, \text { arg }: V)}$ \\
\hline ThrowPoinTsTo (meth $: M$, ctx $: C$, heap $: H$, hctx $: H C)$ \\
\hline
\end{tabular}

Fig. 3. Datalog input and output relations for the exception analysis

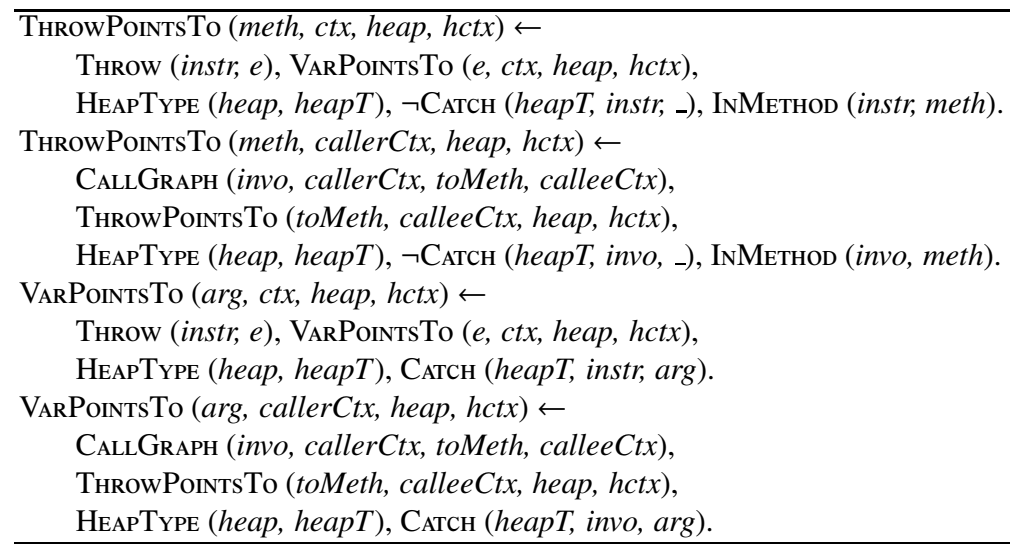

Fig. 4. Datalog rules for the Exception analysis

Figure 3 presents the input and output relations for our analysis. The input relations enhance the language-under-analysis with catch and throw instructions, with Java-like semantics. The Throw (i,e) relation captures throwing at instruction $i$ an expression object that is referenced by local variable $e$. The СATcH $(t, i, a)$ relation connects an instruction $i$ that throws an exception of dynamic type $t$ with the local variable $a$ that will be assigned the exception object at the appropriate catch-site. Although $\mathrm{CATCH}_{\mathrm{AT}}$ does not directly map to intermediate language instructions, one can compute it easily from such 
low-level input. Furthermore, hiding the definition of $\mathrm{CATCH}_{\mathrm{Allows}}$ modeling of exception handlers at different degrees of precision-e.g., a definition of $\mathrm{C}_{\mathrm{ATCH}}$ may or may not consider exception handlers in-order.

Figure 4 shows the exception computation, in mutual recursion with the pointsto analysis. Two syntactic constructs we have not seen before are "_,, meaning "any value", and “ $\neg$ ", signifying negation. The relation we want to compute is THrowPoINTsTo, which captures what exception objects a method may throw at its callers. As can be seen, VARPoINTsTo is used in the definition of ThrowPoInTsTo and vice versa.

\section{Coarsening the Representation of Exceptions}

Although a precise joint points-to and exception analysis algorithm offers significant benefits [1], we next show that there is large room for improvement. The analysis ends up spending much of its time and space computing exception flow. We propose ideas for coarsening the representation of exception objects to address this issue and yield more efficient analyses, without sacrificing precision.

\subsection{Motivation}

Consider the size of the ThrowPointsTo relation for an analysis. This represents the total flow of objects out of methods due to exceptions. For a context-sensitive analysis, this number is a good metric of the work performed by the analysis internally for reasoning about exceptions. It is interesting to compare this number with a similar metric over the VARPoINTsTo relation, namely the subset of VARPoINTsTo facts that concern variables that are either method arguments or return values. This represents the total flow of objects in and out of methods due to normal call and return sequences.

Table 1 shows the results of comparing these two measures for several different analyses: insensitive, call-site sensitive, object-sensitive, and type-sensitive [28], with a context-sensitive heap. The results are over five of the benchmarks in the DaCapo benchmark suite, analyzed with Oracle JDK 1.6. (A full description of our experimental setting can be found in the next section.) Entries with a dash instead of a number did not terminate within the time allotted (90mins).

For the context-insensitive analysis (first results column), the ratio can be understood in intuitive terms: the antlr ratio of 0.22 , for instance, means that, on average, 4.5 times more objects are possibly thrown out of a method than passed into it or returned through regular call and return sequences. This is a counterintuitive result. Human reasoning about how a method interacts with its callers is certainly not dominated by exception objects. Therefore, we see that the joint points-to and exception analysis perhaps pays a disproportionate amount of attention (and expends much effort) on exceptions.

\subsection{Coarse Exceptions}

To reduce the cost of reasoning about exception objects, we propose two simple approaches for coarsening the representation of exception objects. The first is to represent exception objects context-insensitively. This is a rather straightforward idea-even in 
Table 1. Objects on method boundaries compared to exception objects thrown by a method (measured in thousands)

\begin{tabular}{|c|c|c|c|c|c|c|}
\hline & insens & $1 \mathrm{obj}+\mathrm{H}$ & $2 \mathrm{obj}+\mathrm{H}$ & 1type & lcall $+\mathrm{H}$ \\
\hline- & objs passed & 697 & & 10,440 & 3,955 & 17,486 \\
\hline & objs thrown & 3,123 & & 164,392 & 20,783 & 44,118 \\
\hline & ratio & .22 & & .06 & .19 & .40 \\
\hline$=$ & objs passed & $\overline{829}$ & & & 5,681 & 46,952 \\
\hline$\Xi$ & objs thrown & 4,112 & & & 32,905 & 78,593 \\
\hline & ratio & .20 & & & .17 & .60 \\
\hline D & objs passed & 637 & 15,750 & & 6,570 & 18,690 \\
\hline & objs thrown & 4,064 & 138,361 & & 37,634 & 42,140 \\
\hline & ratio & .16 & .11 & & .17 & .44 \\
\hline 朊 & objs passed & $\overline{383}$ & 8,473 & & 3,328 & 8,413 \\
\hline & objs thrown & 2,544 & 60,897 & & 18,928 & 25,297 \\
\hline & ratio & .15 & .14 & & .17 & .33 \\
\hline$=$ & objs passed & 668 & & & 7,480 & 18,895 \\
\hline है & objs thrown & 3,876 & & & 41,351 & 43,376 \\
\hline & ratio & .17 & & & .18 & .44 \\
\hline
\end{tabular}

context-sensitive analyses, several different kinds of objects (e.g., string constants) are more profitably represented context-insensitively. Even before our current work, the Doop framework had the ability to represent exceptions context-insensitively with the right choice of flags. The second approach consists of not just omitting context for exception objects, but also merging the objects themselves, remembering only a single representative per (dynamic) type. That is, all points-to information concerning exception objects is merged "at the source"-all objects of the same type become one.

This is a fitting approach for exception objects because it relies upon intuition on how exception objects are used in practice. Specifically, the intuition is that exception objects have mostly control-flow significance (i.e., they are used as type labels determining what exception handler is to be executed) and little data-flow impact (i.e., the data stored in exception objects' fields do not affect the precision of an overall points-to analysis). In other words, an exception object's dynamic type alone is an excellent approximation of the object itself. Our measurements of the next section show that this is the case.

Figure 5 shows the changes to earlier rules required to implement the two approaches. The original logic of allocating an object is removed and replaced with two cases: if the allocated object is not an instance of an exception type, then the original allocation logic applies. If it is, then the object is allocated context-insensitively (by using a constant context instead of calling the RECORD function to create a new one). Furthermore, in the case of merging exception objects, the object itself is replaced by a representative object of its type (arbitrarily chosen to be the object of the same type with the minimum internal identifier). Note that the definition of an exception type consists of merely looking up all types used in catch clauses- the definition could also be replaced by the weaker condition of whether a type is a subtype of Throwable.

There are some desirable properties of replacing objects with per-type representatives at their creation site. Most importantly, this approach leaves the rest of the analysis unchanged and can maintain all its precision features. Compared to past approaches to 
Commmon core of coarsening logic: object allocation rule is replaced by refined version

CHContext ("ConstantHeapCtx") $\leftarrow$ True.

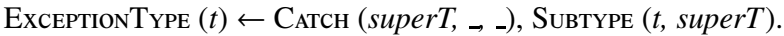

RECORD $($ heap, ctx $)=\boldsymbol{h c t x}$,

VARPointsTo (var, ctw, hctx) $\leftarrow$

Reachable (meth, ctx), Alloc (var, heap, meth).

RECORD (heap, ctx) $=$ hctx,

VARPoInTsTo (var, ctx, heap, hctx) $\leftarrow$

Reachable (meth, ctx), Alloc (var, heap, meth),

HeApType (heap, heapT), ᄀEXCEPTIONTyPE (heapT).

Additional Rule (over common core) for Context-insensitive treatment

VARPoINTsTo (var, ctx, heap, hctx) $\leftarrow$

Reachable (meth, ctx), Alloc (var, heap, meth), HeapType (heap, heapT),

EXCEPTIONTyPE (heapT), CHCONTEXT (hctx).

Additional Rules (over common core) for merging exceptions by use of representative objects

REPRESENTATIVE (heap, reprH) $\leftarrow$

HEAPTYPE $($ heap, heapT $)$, reprHeap $=\min <\operatorname{HEAPTYPE~}($ ?, heapT $)>$.

VARPoINTsTo (var, ctx, reprH, hctx) $\leftarrow$

Reachable (meth, ctx), Alloc (var, heap, meth), HeapType (heap, heapT),

EXceptionType (heapT), CHContext (hctx), Representative (heap, reprH).

Fig. 5. Changes over the rules of Figures 2 and 4 for the two treatments that coarsen the representation of exception objects. The object allocation rule is shown striken out to indicate that it is replaced by a new, conditional version, immediately below. The rules introduce a constant heap context, CHContext, as well as auxiliary relations ExceptionType $(t: T)$ and RePresentative (heap : $H$, reprH $: H$ ).

merging exceptions (e.g., the single-global-variable assignment of Spark or PADDLE) we can maintain all the precision resulting from considering exception handlers in order, filtering caught exceptions, and taking into account the specific instructions under the scope of an exception handler. These have been shown to be important features for the precision and performance of the underlying points-to analysis. Ignoring the order of exception handlers, for instance, results in a much less precise context-sensitive callgraph, with 50\% more edges [1, Fig.13].

\section{Experiments}

We next present the results of our experiments with the two ideas for coarsening the representation of exception objects. As we will see, our approach yields substantial 
performance improvements without sacrificing virtually any precision. This is a rather surprising result. Given how crucial the handling of exceptions has been for the precision of the joint points-to and exception analysis, one would expect that representing exception objects crudely (by merging them per-type) would have serious precision implications. For comparison, Bravenboer and Smaragdakis attempted a different approximation: they represented the ThrowPoINTsTo relation context-insensitively (i.e., by dropping the ctx argument) and found this to significantly hurt the precision of the points-to analysis [1, Sec.5.2], e.g., increasing points-to sets by $10 \% 3$

Our implementation is in the Doop framework and was run on the LogicBlox Datalog engine, v.3.9.0. We use a 64-bit machine with a quad-core Xeon E5530 2.4GHz CPU (only one thread was active at a time) and 24GB of RAM. We analyzed the DaCapo benchmark programs, v.2006-10-MR2 with JDK 1.6.0_30. The choice of JDK is highly significant for Java static analysis. Earlier published Doop results [1, 2, 28] were for JDK 1.4. We chose to present JDK 1.6 results since it is recent, much larger, and more representative of actual use. However, results for JDK 1.4 can also be found in the first author's M.Sc. thesis, available at http://cgi.di.uoa.gr/ gkast/MSc_Thesis.pdf.

There are three primary questions we would like to answer with our experiments:

1. Can we reduce the cost of points-to and exception analysis by coarsening the representation of exception objects, without sacrificing precision?

2. Is the "simple" coarsening approach of treating exceptions context-insensitively sufficient or do we get significant extra benefit from merging exception objects pertype?

3. Do our techniques address the motivation of Table 11 i.e., produce results that roughly match human expectations when reasoning about objects that flow in and out of methods due to exceptions vs. normal call-returns?

Tables 2 and 3 show the time and space savings of the coarsening techniques over a large set of analyses, ranging from context-insensitive to a highly-precise 2-object-sensitive with a 2 -context-sensitive heap $(2 \mathrm{obj}+2 \mathrm{H})$. The analysis variety includes a mix of callsite-, type-, and object-sensitive analyses. Entries with a dash instead of a number are due to analyses that did not terminate within 90 mins. Entries with neither a number nor a dash mean that we did not run the corresponding experiment. (This only happened for experiments on the full-sensitive treatment of exceptions, which we omitted because the main trends were already clear from a smaller subset of our measurements - those for benchmarks and analyses also shown earlier in Table 1.)

As can be seen, the results demonstrate a substantial benefit in the "bottom-line" performance of the joint analysis from representing exception objects coarsely. Furthermore, the simple approach of dealing with exceptions context-insensitively is clearly insufficient. The advantage of merging objects over merely eliding context can be as high as a $3.4 x$ boost in performance, and rarely falls below a $50 \%$ speedup. Space savings tell a similar story, to a lesser but still large extent.

The major question we are addressing next is whether these significant performance improvements entail sacrifices in precision. This requires us to first state the question

\footnotetext{
${ }^{3}$ We repeated several experiments from [1] in our setting for validation but do not report them here since the results are effectively the same as in that publication.
} 
Table 2. Execution time (seconds) for a variety of analyses on various benchmarks

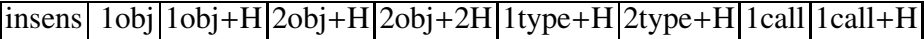

\begin{tabular}{|c|c|c|c|c|c|c|c|c|c|c|}
\hline \multirow{5}{*}{$\exists$} & sens & 120 & 338 & & 3207 & & 543 & 400 & 245 & 975 \\
\hline & insens & 111 & 319 & 1089 & 574 & 2785 & 334 & 209 & 238 & 543 \\
\hline & merge & 75 & 199 & 899 & 249 & 2313 & 217 & 121 & 128 & 420 \\
\hline & \begin{tabular}{|l|} 
sen/ins \\
\end{tabular} & 1.08 & 1.06 & & 5.58 & & 1.62 & 1.91 & 1.02 & 1.79 \\
\hline & ins/mer & 1.48 & 1.60 & 1.21 & 2.30 & 1.20 & 1.53 & 1.72 & 1.85 & 1.29 \\
\hline \multirow{5}{*}{ 告 } & sens & 120 & 1065 & & & & 826 & 1921 & 426 & 3403 \\
\hline & insens & 120 & 1057 & 2337 & - & - & 483 & 553 & 429 & 1795 \\
\hline & merge & 68 & 432 & 1727 & - & - & 292 & 162 & 208 & 1496 \\
\hline & \begin{tabular}{|l|} 
sen/ins \\
\end{tabular} & 1.00 & 1.00 & & - & - & 1.71 & 3.47 & 1.00 & 1.79 \\
\hline & ins/mer & 1.76 & 2.44 & 1.35 & - & - & 1.65 & 3.41 & 2.06 & 1.19 \\
\hline \multirow{4}{*}{ 吾 } & sens & & & & & & & & & \\
\hline & insens & 240 & 2932 & - & & & 1597 & 699 & 591 & 1334 \\
\hline & merge & 138 & 1434 & - & 999 & - & 1253 & 256 & 319 & 1115 \\
\hline & \begin{tabular}{|l|} 
sen/ins \\
ins/mer
\end{tabular} & 1.73 & 2.04 & & & & 1.27 & 2.73 & 1.85 & 1.19 \\
\hline \multirow{5}{*}{ 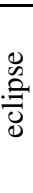 } & sens & 91 & 314 & 2243 & & - & 892 & 800 & 230 & 1099 \\
\hline & insens & 90 & 315 & 800 & 1059 & - & 508 & 348 & 231 & 642 \\
\hline & merge & 52 & 140 & 634 & 623 & - & 269 & 187 & 90 & 500 \\
\hline & \begin{tabular}{|l|}
$\operatorname{sen} /$ ins \\
\end{tabular} & 1.00 & 1.00 & 2.80 & & - & 1.75 & 2.30 & 1.00 & 1.71 \\
\hline & ins/mer & 1.73 & 2.25 & 1.26 & 1.69 & - & 1.88 & 1.86 & 2.56 & 1.28 \\
\hline \multirow{4}{*}{ อี } & sens & & & & & & & & & \\
\hline & insens & 96 & 636 & 2023 & - & - & & & 237 & 613 \\
\hline & merge & 60 & 129 & 944 & - & - & 258 & 768 & 98 & 429 \\
\hline & \begin{tabular}{|l|} 
sen/ins \\
ins/mer
\end{tabular} & 1.60 & 4.93 & 2.14 & -1 & - & - & - & 2.42 & 1.43 \\
\hline \multirow{5}{*}{ 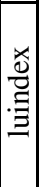 } & \begin{tabular}{|c|} 
sens \\
\end{tabular} & 71 & & 838 & & & 411 & & & 524 \\
\hline & insens & 67 & 168 & 395 & 391 & 2247 & 239 & 168 & 133 & 288 \\
\hline & merge & 45 & 86 & 281 & 153 & 1982 & 142 & 86 & 67 & 195 \\
\hline & \begin{tabular}{|l|} 
sen/ins \\
\end{tabular} & 1.06 & & 2.12 & & & & & & 1.82 \\
\hline & ins/mer & 1.48 & 1.95 & 1.40 & 2.55 & 1.13 & 1.68 & 1.95 & 1.98 & 1.47 \\
\hline \multirow{4}{*}{ 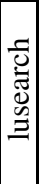 } & sens & & & & & & & & & \\
\hline & insens & 69 & 185 & 428 & 459 & 3024 & 262 & 169 & 145 & 302 \\
\hline & merge & 45 & 97 & 299 & 214 & 2723 & 158 & 87 & 71 & 210 \\
\hline & \begin{tabular}{|l|} 
sen/ins \\
ins/mer
\end{tabular} & 1.53 & 1.90 & 1.43 & 2.14 & 1.11 & 1.66 & 1.94 & 2.04 & 1.43 \\
\hline \multirow{3}{*}{ ב्ञ } & $\begin{array}{l}\text { sens } \\
\text { insens }\end{array}$ & 105 & 274 & 567 & 427 & 2330 & 327 & 213 & 181 & 392 \\
\hline & merge & 66 & 153 & 379 & 188 & 2076 & 207 & 129 & 100 & 280 \\
\hline & \begin{tabular}{|l|} 
sen/ins \\
ins/mer
\end{tabular} & 159 & 179 & 149 & 2.27 & 112 & 157 & 165 & 181 & 140 \\
\hline \multirow{5}{*}{ 胥 } & sens & 116 & & & & & 1091 & & & 1214 \\
\hline & insens & 118 & 463 & 1139 & & & 579 & 349 & 249 & 672 \\
\hline & merge & 70 & 219 & 836 & - & - & 470 & 200 & 126 & 521 \\
\hline & \begin{tabular}{|l|} 
sen/ins \\
\end{tabular} & 1.00 & & & & & 1.88 & & & 1.80 \\
\hline & ins/mer & 1.68 & 2.11 & 1.36 & & & 1.23 & 1.74 & 1.97 & 1.29 \\
\hline
\end{tabular}


Table 3. Disk footprint (MB) for a variety of analyses on various benchmarks

\begin{tabular}{|c|c|c|c|c|c|c|c|c|c|c|}
\hline & & insens & 1obj & $1 \mathrm{obj}+\mathrm{H}$ & $2 \mathrm{obj}+\mathrm{H}$ & $2 \mathrm{obj}+2 \mathrm{H}$ & 1type+H & 2 type $+\mathrm{H}$ & 1 call & 1call+H \\
\hline \multirow{5}{*}{$\bar{\Xi}$} & sens & 649 & 996 & & 4608 & & 1433 & 1228 & 945 & 3174 \\
\hline & insens & 649 & 996 & 2560 & 1536 & 3686 & 963 & 735 & 945 & 1740 \\
\hline & merge & 544 & 683 & 2048 & 978 & 3072 & 675 & 518 & 661 & 1433 \\
\hline & \begin{tabular}{|l|} 
sen/ins \\
\end{tabular} & $\overline{1.00}$ & 1.00 & & 3.00 & & 1.48 & 1.67 & \begin{tabular}{|l|}
1.00 \\
\end{tabular} & 1.82 \\
\hline & ins/mer & 1.19 & 1.45 & 1.25 & 1.57 & 1.19 & 1.42 & 1.41 & 1.42 & 1.21 \\
\hline \multirow{5}{*}{$\overline{\tilde{z}}$} & sens & 460 & 1126 & & & & 1433 & 2150 & 1228 & 5120 \\
\hline & insens & 461 & 1126 & 2457 & - & & 923 & 992 & 1228 & 3379 \\
\hline & merge & 363 & 663 & 1843 & & & 586 & 505 & 773 & 2969 \\
\hline & \begin{tabular}{|l|} 
sen/ins \\
\end{tabular} & $\overline{1.00}$ & 1.00 & & & & 1.55 & 2.16 & 1.00 & 1.51 \\
\hline & ins/mer & 1.26 & 1.69 & 1.33 & - & & 1.57 & 1.96 & 1.58 & 1.13 \\
\hline \multirow{3}{*}{ है } & $\begin{array}{c}\text { sens } \\
\text { insens }\end{array}$ & 968 & 3072 & - & & & 2764 & 1536 & 1945 & 3270 \\
\hline & merge & 653 & 1740 & & & & 1945 & 811 & 1331 & 2560 \\
\hline & \begin{tabular}{|l|} 
sen/ins \\
ins/mer
\end{tabular} & 1.48 & 1.76 & & & & 1.42 & 1.89 & 1.46 & 1.27 \\
\hline \multirow{5}{*}{0} & sens & $\overline{428}$ & 748 & 4505 & & & 2048 & 1945 & 694 & 2867 \\
\hline & insens & 429 & 748 & 2048 & 2252 & & 1433 & 1012 & 694 & 1638 \\
\hline & merge & 300 & 405 & 1433 & 1536 & - & 679 & 602 & 444 & 1433 \\
\hline & sen/ins & $\overline{1.00}$ & 1.00 & 2.20 & & & 1.43 & 1.92 & 1.00 & 1.75 \\
\hline & ins/mer & 1.43 & 1.84 & 1.42 & 1.46 & & 2.11 & 1.68 & 1.56 & 1.14 \\
\hline \multirow{4}{*}{ 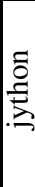 } & sens & & & & & & & & & \\
\hline & insens & 604 & 1228 & 3584 & & & & & 980 & 1740 \\
\hline & merge & 472 & 609 & 2355 & & & 798 & 1740 & 601 & 1331 \\
\hline & \begin{tabular}{|l|} 
sen/ins \\
ins/mer
\end{tabular} & 1.27 & 2.01 & 1.52 & & & - & - & 1.63 & 1.30 \\
\hline \multirow{5}{*}{ 离 } & sens & $\overline{346}$ & & 2252 & & & 929 & & & 1638 \\
\hline & insens & 346 & 496 & 1126 & 1126 & 2764 & 645 & 549 & 508 & 874 \\
\hline & merge & 265 & 317 & 716 & 565 & 2150 & 399 & 327 & 349 & 684 \\
\hline & \begin{tabular}{|l|} 
sen/ins \\
\end{tabular} & $\overline{1.00}$ & & 2.00 & & & & & & 1.87 \\
\hline & ins/mer & 1.30 & 1.56 & 1.57 & 1.99 & 1.28 & 1.62 & 1.67 & 1.45 & 1.27 \\
\hline \multirow{4}{*}{ 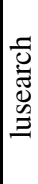 } & sens & & & & & & & & & \\
\hline & insens & 367 & 517 & 1228 & 1228 & 3379 & 681 & 550 & 548 & 926 \\
\hline & merge & 277 & 334 & 723 & 690 & 2867 & 424 & 327 & 370 & 722 \\
\hline & \begin{tabular}{|l|} 
sen/ins \\
ins/mer
\end{tabular} & 1.32 & 1.54 & 1.69 & 1.77 & 1.17 & 1.60 & 1.68 & 1.48 & 1.28 \\
\hline \multirow{3}{*}{ है } & $\begin{array}{c}\text { sens } \\
\text { insens }\end{array}$ & 602 & 817 & 1536 & 1331 & 2969 & 948 & 785 & 814 & 1331 \\
\hline & merge & 505 & 585 & 1017 & 839 & 2457 & 647 & 560 & 631 & 1126 \\
\hline & \begin{tabular}{|l|} 
sen/ins \\
ins/mer
\end{tabular} & 1.19 & 1.39 & 1.51 & 1.58 & 1.20 & 1.46 & 1.40 & $\mid 1.29$ & 1.18 \\
\hline \multirow{5}{*}{ 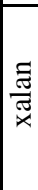 } & sens & $\overline{614}$ & & & & & 2457 & & & 2969 \\
\hline & insens & 614 & 1331 & 3174 & & & 1638 & 1126 & 940 & 1843 \\
\hline & merge & 487 & 710 & 2252 & & - & 823 & 684 & 659 & 1536 \\
\hline & sen/ins & $\mathbf{1 . 0 0}$ & & & & & 1.50 & & & 1.61 \\
\hline & ins/mer & 1.26 & 1.87 & 1.41 & & & 2.00 & 1.64 & 1.42 & 1.20 \\
\hline
\end{tabular}


Table 4. Metrics concerning precision for a variety of analyses. Jython omitted for space.

\begin{tabular}{|c|c|c|c|c|c|c|c|c|}
\hline & edges & meths & V-calls & poly v-calls & casts & fail casts \\
\hline \multirow{12}{*}{ 言 } & & sens & & & & & & \\
\hline & & insens & 59075 & 8886 & 33467 & 1924 & 1767 & 985 \\
\hline & & merge & 59075 & 8886 & 33467 & 1924 & 1767 & 985 \\
\hline & & sens & 55445 & 8714 & 32976 & 1712 & 1709 & 611 \\
\hline & & insens & 55445 & 8714 & 32976 & 1712 & 1709 & 611 \\
\hline & & merge & 55445 & 8714 & 32976 & 1712 & 1709 & 611 \\
\hline & & sens & 59738 & 8916 & 33507 & 1948 & 1770 & 1070 \\
\hline & & insens & 59738 & 8916 & 33507 & 1948 & 1770 & 1070 \\
\hline & & merge & 59738 & 8916 & 33507 & 1948 & 1770 & 1070 \\
\hline & & sens & 60797 & 8961 & 33631 & 1985 & 1778 & 1037 \\
\hline & & insens & 60797 & 8961 & 33631 & 1985 & 1778 & 1037 \\
\hline & & merge & 60797 & 8961 & 33631 & 1985 & 1778 & 1037 \\
\hline \multirow{9}{*}{ 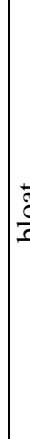 } & & sens & & & & & & \\
\hline & & insens & 65672 & 10116 & 31049 & 2067 & 2815 & 1911 \\
\hline & & merge & 65672 & 10116 & 31049 & 2067 & 2815 & 1911 \\
\hline & & sens & & & & & & \\
\hline & & insens & & & - & & & \\
\hline & & merge & & & - & & & \\
\hline & & sens & 66697 & 10150 & 31089 & 2137 & 2818 & 2045 \\
\hline & & insens & 66697 & 10150 & 31089 & 2137 & 2818 & 2045 \\
\hline & & merge & 66697 & 10150 & 31089 & 2137 & 2818 & 2045 \\
\hline & & sens & 70340 & 10200 & 31214 & 2129 & 2829 & 2007 \\
\hline & & insens & 70340 & 10200 & 31214 & 2129 & 2829 & 2007 \\
\hline & & merge & 70340 & 10200 & 31214 & 2129 & 2829 & 2007 \\
\hline \multirow{11}{*}{ है } & & sens & & & & & & \\
\hline & & 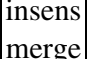 & & & & & & \\
\hline & & sens & & & & & & \\
\hline & & insens & & & & & & \\
\hline & & merge & 59027 & 12510 & 31111 & 1610 & 2765 & 1055 \\
\hline & & sens & & & & & & \\
\hline & & insens & 79871 & 16044 & 39462 & 2725 & 3858 & 2445 \\
\hline & & merge & 79896 & 16044 & 39462 & 2730 & 3858 & 2450 \\
\hline & & sens & & & & & & \\
\hline & & |insens & 81865 & 16134 & 39724 & 2887 & 3887 & 2480 \\
\hline & & merge & 81890 & 16134 & 39724 & 2892 & 3887 & 2485 \\
\hline \multirow{12}{*}{8} & & sens & 49279 & 9408 & 23505 & 1386 & 1984 & 1092 \\
\hline & & insens & 49279 & 9408 & 23505 & 1386 & 1984 & 1092 \\
\hline & & merge & 49282 & 9408 & 23505 & 1386 & 1984 & 1092 \\
\hline & & sens & & & & & & \\
\hline & & insens & 44792 & 9188 & 22852 & 1168 & 1912 & 729 \\
\hline & & merge & 44795 & 9188 & 22852 & 1168 & 1912 & 729 \\
\hline & & sens & 51161 & 9452 & 23634 & 1438 & 1987 & 1198 \\
\hline & $-x^{2}$ & insens & 51161 & 9452 & 23634 & 1438 & 1987 & 1198 \\
\hline & & merge & 51162 & 9452 & 23634 & 1438 & 1987 & 1198 \\
\hline & & sens & 52800 & 9511 & 23716 & 1507 & 2000 & 1154 \\
\hline & & insens & 52800 & 9511 & 23716 & 1507 & 2000 & 1154 \\
\hline & & merge & 52800 & 9511 & 23716 & 1507 & 2000 & 1154 \\
\hline
\end{tabular}


Table 5. Metrics concerning precision for a variety of analyses (cont'd from Table 4)

\begin{tabular}{|c|c|c|c|c|c|c|c|c|}
\hline & \multirow{2}{*}{\begin{tabular}{|l|} 
edges \\
40004
\end{tabular}} & meths & v-calls & poly v-calls & casts & fail casts \\
\hline \multirow{12}{*}{$\stackrel{x}{g}$} & & sens & & 7876 & 18263 & 1110 & 1521 & 796 \\
\hline & & insens & 40004 & 7876 & 18263 & 1110 & 1521 & 796 \\
\hline & & merge & 40004 & 7876 & 18263 & 1110 & 1521 & 796 \\
\hline & & ens & & & & & & \\
\hline & & insens & 36477 & 7702 & 17748 & 899 & 1463 & 496 \\
\hline & & merge & 36477 & 7702 & 17748 & 899 & 1463 & 496 \\
\hline & & sens & 40646 & 7906 & 18303 & 1138 & 1524 & 885 \\
\hline & & insens & 40646 & 7906 & 18303 & 1138 & 1524 & 88 \\
\hline & & merge & 40646 & 7906 & 18303 & 1138 & 1524 & 896 \\
\hline & & sens & 41790 & 7953 & 18492 & 1171 & 1532 & 837 \\
\hline & & insens & 41790 & 7953 & 18492 & 1171 & 1532 & 837 \\
\hline & & merge & 41790 & 7953 & 18492 & 1171 & 1532 & 837 \\
\hline \multirow{10}{*}{$\Xi$} & & $\begin{array}{c}\text { sens } \\
\text { insens }\end{array}$ & 42977 & 8526 & 1556 & 1289 & 1622 & 81 \\
\hline & & merge & 42977 & 8526 & 19556 & 1289 & 1622 & 812 \\
\hline & & sens & & & & & & \\
\hline & & insens & 39352 & 8344 & 19048 & 1071 & 1564 & 508 \\
\hline & & merge & 39352 & 8344 & 19048 & 1071 & 1564 & 508 \\
\hline & & sens & & & & & & \\
\hline & & insens & 43676 & 8558 & 19620 & 1319 & 1625 & 927 \\
\hline & & merge & 43676 & 8558 & 19620 & 1319 & 1625 & 934 \\
\hline & & $\begin{array}{c}\text { sens } \\
\text { insens }\end{array}$ & 45071 & 8626 & 9857 & 1352 & 1643 & 938 \\
\hline & & merge & 45071 & 8626 & 19857 & 1352 & 1643 & 938 \\
\hline & & $\begin{array}{l}\text { sens } \\
\text { insens }\end{array}$ & 826 & 9277 & 591 & 1168 & 990 & 21 \\
\hline & & merge & 46826 & 9277 & 21591 & 1168 & $\begin{array}{l}1990 \\
1990\end{array}$ & $\begin{array}{l}121 \\
121\end{array}$ \\
\hline & & ens & & & & & & \\
\hline & & insens & 42988 & 9090 & 21004 & 942 & 1,931 & 840 \\
\hline & & merge & 42988 & 9090 & 21004 & 942 & 1,931 & 84 \\
\hline & & sens & & & & & & \\
\hline & & insens & 47539 & 9311 & 21632 & 1192 & 1993 & 1311 \\
\hline & & merge & 47540 & 9311 & 21632 & 1192 & 1993 & $131^{\prime}$ \\
\hline & & $\begin{array}{c}\text { sens } \\
\text { insens }\end{array}$ & 48895 & 9371 & 1843 & 1240 & 2003 & 1273 \\
\hline & $y$ & merge & 48895 & 9371 & 21843 & 1240 & 2003 & 1273 \\
\hline & & sens & & & & & & \\
\hline & & insens & 54033 & 10511 & 25683 & 1857 & 2042 & 1055 \\
\hline & & merge & 54038 & 10511 & 25683 & 1858 & 2042 & 1055 \\
\hline & & ens & & & & & & \\
\hline & $\nu^{0}$ & ens & & & & & & \\
\hline & & sens & 54792 & 10561 & 25760 & 1887 & 2049 & 1235 \\
\hline & & insens & 54792 & 10561 & 25760 & 1887 & 2049 & 1235 \\
\hline & & merge & 54796 & 10561 & 25760 & 1888 & 2049 & 1235 \\
\hline & & sens & 56658 & 10613 & 25891 & 1966 & 2059 & $\overline{1203}$ \\
\hline & & insens & 56658 & 10613 & 25891 & 1966 & 2059 & 1203 \\
\hline & & merge & 56666 & 10613 & 25891 & 1967 & 2059 & 120 \\
\hline
\end{tabular}


Table 6. Objects on method boundaries compared to exception objects thrown by a method (measured in thousands)

\begin{tabular}{|c|c|c|c|c|c|c|c|c|c|c|c|}
\hline & \multicolumn{2}{|c|}{ insens } & \multicolumn{2}{|c|}{$1 \mathrm{obj}+\mathrm{H}$} & \multicolumn{2}{|c|}{$2 \mathrm{obj}+\mathrm{H}$} & \multicolumn{2}{|c|}{ 1type $+\mathrm{H}$} & \multicolumn{2}{|c|}{ 1call $+\mathrm{H}$} \\
\hline & & insens & merge & insens & merge & sens & merge & insens & merge & insens & merge \\
\hline \multirow[t]{3}{*}{ 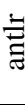 } & objs p & 697 & 651 & 26,867 & 25,271 & 6,983 & 6,079 & 4,702 & 4,528 & 17,236 & 17,15 \\
\hline & objs t & 3,123 & 204 & 14,197 & 965 & 23,856 & 1,730 & 7,084 & 428 & 12,461 & 822 \\
\hline & 12 & .22 & 3.18 & 1.89 & 26.16 & .29 & 3.51 & .66 & 10.56 & 1.38 & 20.86 \\
\hline \multirow{3}{*}{$\pi$} & js & 829 & 781 & 22,633 & 22,325 & & & 5,338 & 5,167 & 46,650 & $\overline{46,563}$ \\
\hline & objs & 4,112 & 257 & 18,697 & 1,189 & & & 10,140 & 589 & 20,048 & 1,251 \\
\hline & rate & .20 & 3.02 & 1.21 & 18.76 & & & .52 & 8.76 & 2.32 & 37.22 \\
\hline \multirow{3}{*}{$\exists$} & objs & 315 & 1,723 & & & & 16,739 & & 18,721 & 46,158 & $\overline{41,909}$ \\
\hline & objs & 331 & 414 & & & & 7,231 & & 1,357 & 19,747 & 1,371 \\
\hline & ra & .27 & 4.15 & & & & 2.31 & & 13.79 & 2.33 & 30.56 \\
\hline \multirow{3}{*}{$\begin{array}{l}0 \\
\stackrel{0}{0} \\
\text { : } \\
\end{array}$} & js & 637 & 539 & $\overline{14,045}$ & 16,875 & 15,234 & 14,357 & 6,025 & 6,471 & 18,311 & 18,18 \\
\hline & objs & ,064 & 248 & ,983 & 047 & 6,699 & 2,612 & 0,516 & 593 & 11,829 & 777 \\
\hline & ratio & .15 & 2.17 & .87 & 16.10 & .41 & 5.49 & .57 & 10.90 & 1.54 & 23.40 \\
\hline \multirow[b]{3}{*}{$\approx$} & $\overline{\mathrm{d}}$ & 801 & $\overline{479}$ & 43,068 & 35,106 & & & & & 19,307 & \\
\hline & objs & 452 & 215 & 20,449 & 25 & & & & & 11,288 & \\
\hline & ratio & .23 & 2.23 & 2.10 & 34.21 & & & & & 1.71 & \\
\hline \multirow{6}{*}{ 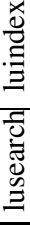 } & objs 1 & 383 & 339 & 7,664 & 7,414 & 3,525 & 3,037 & 3,031 & 2,881 & 8,186 & $\overline{8,109}$ \\
\hline & objs & 2,544 & 166 & 8,953 & 606 & 21,401 & 1,521 & 6,055 & 363 & 7,218 & 486 \\
\hline & ratio & .15 & 2.03 & .85 & 12.21 & .16 & 1.99 & .50 & 7.92 & 1.13 & 16.67 \\
\hline & objs passed & 429 & 385 & 8,085 & 7,822 & 4,519 & 4,010 & 3,319 & 3,165 & 9,005 & $\overline{8,928}$ \\
\hline & objs & 2,764 & 180 & 9,222 & 626 & 19,432 & 1,435 & 6,341 & 379 & 7,880 & 528 \\
\hline & ratio & .15 & 2.13 & .87 & 12.49 & .23 & 2.79 & .52 & 8.33 & 1.14 & 16.90 \\
\hline \multirow{3}{*}{ ב } & objs passed & 461 & 414 & 9,273 & 8,949 & 4,388 & 3,864 & 3,500 & 3,337 & 10,948 & 10,867 \\
\hline & objs t & 3,008 & 198 & 10,409 & 695 & 19,961 & 1,465 & 7,100 & 431 & 8,529 & 577 \\
\hline & & .15 & 2.09 & .89 & 12.87 & .21 & 2.63 & .49 & 7.73 & 1.28 & 18.81 \\
\hline \multirow{3}{*}{$\frac{\Xi}{\tilde{\Xi}}$} & objs passed & 668 & 589 & 24,618 & 24,155 & & & 6,350 & 5,821 & 18,448 & $\overline{18,301}$ \\
\hline & js t & 3,876 & 251 & 20,765 & 1,372 & & & 11,278 & 641 & 12,198 & 810 \\
\hline & & .17 & 2.34 & 1.18 & 17.60 & & & .5 & 9.08 & 1.51 & 22.5 \\
\hline
\end{tabular}

appropriately. Since all exception objects of the same type are merged, it makes little sense to query points-to information for variables holding such objects (e.g., local variables inside exception handlers). Every such variable will appear to spuriously point to any object of the same dynamic type. Instead, what we want to do is examine the impact of merging exception objects on the rest of the points-to analysis. That is, a client analysis that cares about exception objects themselves should not employ our techniques. The question, however, is whether an analysis that applies over the whole program will be affected by the coarse exception representations.

Tables 4 and 5 show precision metrics for our benchmarks and a subset (for space reasons) of our analyses. We show the size of the computed call-graph, in terms of both nodes (i.e., methods) and edges, as well as the number of virtual calls that cannot be statically resolved and casts that cannot be statically be proven safe. (The total number of reachable virtual calls and casts are given for reference.) From our past experience, the call-graph metrics are generally excellent proxies for the overall precision of an analysis, and even tiny changes are reflected on them. 
As can be seen, the precision of the program analysis remains virtually unaffected by the coarse representations of exceptions. This confirms that our merged exception objects still carry the essence of the information that the rest of the program needs from them.

The final question from our experiments is whether these two techniques address the motivating measurements of Section 3.1. Table 6 shows the same metrics of (contextsensitive) objects passed to/from methods vs. thrown for the analysis using our coarse representations of exceptions. As can be seen, the handling of exceptions contextinsensitively does not suffice to bring the relative ratio of the metrics close to expected values, but merging exception objects per-type does. Specifically, for all values of the "merge" column, the total number of objects passed via calls and returns is several times higher than the number of objects potentially thrown. Thus, the analysis is allocating its reasoning effort in a way that closely matches what one would expect intuitively, possibly indicating that large further improvements are unlikely.

\section{Related Work}

We next discuss in more detail some of the past work in points-to analysis combined with exceptions.

As mentioned earlier, points-to analysis frameworks SPARK [19] and PAdDLE [18, 20] both use imprecise exception analysis via assignment of thrown exceptions to a single global variable. Even if this were to change to distinct per-type variables, it would still have significant precision shortcomings compared to our approach since the order and scope of exception handlers would be ignored. The Soot framework also has a separate exception analysis [16] that is not based on a pointer analysis.

The IBM Research WALA [6] static analysis library supports several different pointer analysis configurations. The points-to analyses of WALA support computing which exceptions a method can throw (analogously to our THrowPoINTsTo relation), but no results of WALA's accuracy or speed have been reported in the literature. WaLA allows an exception object to be represented by-type (analogously to our coarsening) but it is unclear how the underlying analysis compares to our joint exception and points-to analysis. It will be interesting to compare our analyses to WALA in future work.

Type-based approaches to dealing with exception objects have also been explored before [14, 15], in the context of a separate exception analysis (i.e., not jointly with a precise points-to analysis and not in comparison to an object-based exception representation).

$b d d b d d b$ is a Datalog and BDD-based database that has been employed for points-to analysis [29, 30]. The publications do not discuss exception analysis, yet the bddbddb distribution examples do propagate exceptions over the control-flow graph. One of the differences between Doop and bddbddb is that Doop expresses the entire analysis in Datalog and only relies on basic input facts. In contrast, the points-to analyses of bddbddb largely rely on pre-computed input facts, such as a call-graph, reducing the Datalog analysis to just a few lines of code for propagating points-to data. For exception analysis, bddbddb ignores the order of exception handlers and also disables filtering of caught exceptions. Both of these features are crucial for precision. 
Chatterjee et al. analyze the worst-case complexity of fully-precise pointer analysis with exceptions [3]. This is a theoretical analysis with no current application to practical points-to algorithms.

Sinha et al. discuss how to represent exception flow in the control-flow graph [27]. One of the topics is handling finally clauses. We analyze Java bytecode, hence the complex control-flow of finally clauses is already handled by the Java compiler.

Choi et al. suggested a compact intraprocedural control-flow representation that collapses the large number of edges to exceptions handlers [4]. Our analyses are interprocedural and flow-insensitive, so not directly comparable to that work.

\section{Conclusions}

When analyzing an object-oriented program, exceptions pose an interesting challenge. If completely ignored, valuable properties of the program are lost and large amounts of code appear unexercised. If handled in isolation (either before or after points-to analysis) the result is imprecise and the analysis suffers from inefficiency. A joint points-to and exception analysis offers the answer but has significant time and space cost due to the precise representation of exception objects. We showed that we can profitably coarsen the representation of exception objects in such a joint analysis. Precision remains unaffected, for the parts of the analysis not directly pertaining to exception objects, i.e., for most common analysis clients, such as cast elimination, devirtualization, and call-graph construction. At the same time, performance is significantly enhanced. Thus the approach is a clear win and is now the default policy for exception handling in the Doop framework.

There are interesting avenues for further work along the directions of the paper. Our approach is based on standard patterns of use of exception objects. These patterns can perhaps be generalized to other kinds of objects used as "message carriers". Furthermore, the question arises of how such patterns translate across languages. Is there an analogous concept in functional languages that can be exploited to gain scalability? Also, it remains to be seen whether similar approaches can apply to alias analysis in $\mathrm{C}++$ in the presence of exceptions.

Acknowledgments. We gratefully acknowledge funding by the European Union under a Marie Curie International Reintegration Grant and a European Research Council Starting/Consolidator grant. We also gratefully acknowledge the support of the Greek Secretariat for Research and Technology under an Excellence (Aristeia) award. The work in this paper was significantly aided by pre-existing mechanisms that Martin Bravenboer has implemented in the Doop framework.

\section{References}

1. Bravenboer, M., Smaragdakis, Y.: Exception analysis and points-to analysis: Better together. In: Dillon, L. (ed.) ISSTA 2009: Proceedings of the 2009 International Symposium on Software Testing and Analysis, New York, NY, USA (July 2009) 
2. Bravenboer, M., Smaragdakis, Y.: Strictly declarative specification of sophisticated points-to analyses. In: OOPSLA 2009: 24th Annual ACM SIGPLAN Conference on Object Oriented Programming, Systems, Languages, and Applications. ACM, New York (2009)

3. Chatterjee, R., Ryder, B.G., Landi, W.A.: Complexity of points-to analysis of Java in the presence of exceptions. IEEE Trans. Softw. Eng. 27(6), 481-512 (2001)

4. Choi, J.D., Grove, D., Hind, M., Sarkar, V.: Efficient and precise modeling of exceptions for the analysis of Java programs. SIGSOFT Softw. Eng. Notes 24(5), 21-31 (1999)

5. Eichberg, M., Kloppenburg, S., Klose, K., Mezini, M.: Defining and continuous checking of structural program dependencies. In: ICSE 2008: Proc. of the 30th Int. Conf. on Software Engineering, pp. 391-400. ACM, New York (2008)

6. Fink, S.J., et al.: T.J. Watson libraries for analysis (WALA), http://wala.sourceforge.net

7. Fu, C., Milanova, A., Ryder, B.G., Wonnacott, D.G.: Robustness testing of Java server applications. IEEE Trans. Softw. Eng. 31(4), 292-311 (2005)

8. Fu, C., Ryder, B.G.: Exception-chain analysis: Revealing exception handling architecture in Java server applications. In: ICSE 2007: Proceedings of the 29th International Conference on Software Engineering, pp. 230-239. IEEE Computer Society, Washington, DC (2007)

9. Fu, C., Ryder, B.G., Milanova, A., Wonnacott, D.: Testing of Java web services for robustness. In: ISSTA 2004: Proceedings of the 2004 ACM SIGSOFT International Symposium on Software Testing and Analysis, pp. 23-34. ACM, New York (2004)

10. Guarnieri, S., Livshits, B.: GateKeeper: mostly static enforcement of security and reliability policies for Javascript code. In: Proceedings of the 18th USENIX Security Symposium, SSYM 2009, pp. 151-168. USENIX Association, Berkeley (2009), http://dl.acm.org/citation. cfm?id=1855768.1855778

11. Hajiyev, E., Verbaere, M., de Moor, O.: codeQuest: Scalable Source Code Queries with Datalog. In: Thomas, D. (ed.) ECOOP 2006. LNCS, vol. 4067, pp. 2-27. Springer, Heidelberg (2006)

12. Hardekopf, B., Lin, C.: The ant and the grasshopper: fast and accurate pointer analysis for millions of lines of code. In: PLDI 2007: Proc. ACM SIGPLAN Conf. on Programming Language Design and Implementation, pp. 290-299. ACM, New York (2007)

13. Hardekopf, B., Lin, C.: Semi-sparse flow-sensitive pointer analysis. In: POPL 2009: Proceedings of the 36th Annual ACM SIGPLAN-SIGACT Symposium on Principles of Programming Languages, pp. 226-238. ACM, New York (2009)

14. Jo, J.-W., Chang, B.-M.: Constructing Control Flow Graph for Java by Decoupling Exception Flow from Normal Flow. In: Laganá, A., Gavrilova, M.L., Kumar, V., Mun, Y., Tan, C.J.K., Gervasi, O. (eds.) ICCSA 2004. LNCS, vol. 3043, pp. 106-113. Springer, Heidelberg (2004)

15. Jo, J.W., Chang, B.M., Yi, K., Choe, K.M.: An uncaught exception analysis for Java. Journal of Systems and Software 72(1), 59-69 (2004)

16. Jorgensen, J.: Improving the precision and correctness of exception analysis in Soot. Tech. Rep. 2003-3, McGill University (September 2004)

17. Lam, M.S., Whaley, J., Livshits, V.B., Martin, M.C., Avots, D., Carbin, M., Unkel, C.: Context-sensitive program analysis as database queries. In: PODS 2005: Proc. of the TwentyFourth ACM SIGMOD-SIGACT-SIGART Symposium on Principles of Database Systems, pp. 1-12. ACM, New York (2005)

18. Lhoták, O.: Program Analysis using Binary Decision Diagrams. Ph.D. thesis, McGill University (January 2006)

19. Lhoták, O., Hendren, L.: Scaling Java Points-to Analysis Using SPARK. In: Hedin, G. (ed.) CC 2003. LNCS, vol. 2622, pp. 153-169. Springer, Heidelberg (2003)

20. Lhoták, O., Hendren, L.: Evaluating the benefits of context-sensitive points-to analysis using a BDD-based implementation. ACM Trans. Softw. Eng. Methodol. 18(1), 1-53 (2008) 
21. Madsen, M., Livshits, B., Fanning, M.: Practical static analysis of Javascript applications in the presence of frameworks and libraries. Tech. Rep. MSR-TR-2012-66, Microsoft Research (Jully 2012)

22. Might, M., Smaragdakis, Y., Van Horn, D.: Resolving and exploiting the k-CFA paradox: Illuminating functional vs. object-oriented program analysis. In: Conf. on Programming Language Design and Implementation (PLDI), pp. 305-315. ACM (June 2010)

23. Milanova, A., Rountev, A., Ryder, B.G.: Parameterized object sensitivity for points-to analysis for Java. ACM Trans. Softw. Eng. Methodol. 14(1), 1-41 (2005)

24. Reps, T.: Demand interprocedural program analysis using logic databases. In: Ramakrishnan, R. (ed.) Applications of Logic Databases, pp. 163-196. Kluwer Academic Publishers (1994)

25. Sharir, M., Pnueli, A.: Two approaches to interprocedural data flow analysis. In: Muchnick, S.S., Jones, N.D. (eds.) Program Flow Analysis, pp. 189-233. Prentice-Hall, Inc., Englewood Cliffs (1981)

26. Shivers, O.: Control-Flow Analysis of Higher-Order Languages. Ph.D. thesis, Carnegie Mellon University (May 1991)

27. Sinha, S., Harrold, M.J.: Analysis and testing of programs with exception handling constructs. IEEE Trans. Softw. Eng. 26(9), 849-871 (2000)

28. Smaragdakis, Y., Bravenboer, M., Lhoták, O.: Pick your contexts well: Understanding objectsensitivity (the making of a precise and scalable pointer analysis). In: ACM Symposium on Principles of Programming Languages (POPL), pp. 17-30. ACM Press (January 2011)

29. Whaley, J., Avots, D., Carbin, M., Lam, M.S.: Using Datalog with Binary Decision Diagrams for Program Analysis. In: Yi, K. (ed.) APLAS 2005. LNCS, vol. 3780, pp. 97-118. Springer, Heidelberg (2005)

30. Whaley, J., Lam, M.S.: Cloning-based context-sensitive pointer alias analysis using binary decision diagrams. In: PLDI 2004: Proc. of the ACM SIGPLAN 2004 Conf. on Programming Language Design and Implementation, pp. 131-144. ACM, New York (2004) 